\title{
Nonconvex Proximal Incremental Aggregated Gradient Method with Linear Convergence
}

\author{
Wei Peng $^{1}$ • Hui Zhang ${ }^{1}$ • Xiaoya Zhang $^{1}$
}

Received: date / Accepted: date

\begin{abstract}
In this paper, we study the proximal incremental aggregated gradient(PIAG) algorithm for minimizing the sum of L-smooth nonconvex component functions and a proper closed convex function. By exploiting the Lsmooth property and with the help of an error bound condition, we can show that the PIAG method still enjoys some nice linear convergence properties even for nonconvex minimization. To illustrate this, we first demonstrate that the generated sequence globally converges to the stationary point set. Then, there exists a threshold such that the objective function value sequence and the iterate point sequence are R-linearly convergent when the stepsize is chosen below this threshold.
\end{abstract}

Keywords Linear convergence · Nonconvex · Incremental aggregated gradient

\section{Introduction}

A fundamental optimization model emerges in numerous problems including machine learning, signal processing, image science, communication systems, and distributed optimization. Typically, the model is to minimize the sum of $N$ differentiable functions $f_{i}$ which are possibly nonconvex and a convex

\footnotetext{
Wei Peng

E-mail: weipeng0098@126.com

Hui Zhang(Corresponding author)

E-mail: h.zhang1984@163.com

Xiaoya Zhang

E-mail: zhangxiaoya09@nudt.edu.cn

${ }^{1}$ Department of Mathematics, National University of Defense Technology
} 
nonsmooth function $h$ :

$$
\min _{x \in \mathbb{R}^{d}} F(x):=\sum_{i=1}^{N} f_{i}(x)+h(x) .
$$

This problem often arises in large-scale, distributed, parallel optimization subfields with large $N$. Directly computing $\sum_{i=1}^{N} \nabla f_{i}\left(x_{k}\right)$ in the popular forwardbackward splitting (FBS) $[6$ scheme might be prohibitive for large $N$. Thereby a natural method to approximate the gradient of $f(x)$ embedding into FBS scheme is proposed, named as the proximal incremental aggregated gradient (PIAG) method. The key idea of PIAG is to construct an "inexact gradient" $g_{k}$ to substitute the "exact" $\sum_{i=1}^{N} \nabla f\left(x_{k}\right)$. PIAG method is the iterative procedure of three steps:

$$
\begin{aligned}
& g_{k}=\sum_{i=1}^{N} \nabla f_{i}\left(x_{k-\tau_{k}^{i}}\right), \\
& y_{k}=x_{k}-\alpha \cdot g_{k}, \\
& x_{k+1}=\arg \min _{x \in \mathbb{R}^{d}}\left\{h(x)+\frac{1}{2 \alpha}\left\|x-y_{k}\right\|^{2}\right\},
\end{aligned}
$$

where $\tau_{k}^{i}$ are some nonnegative integers representing delayed iterations. In addition, we assume that $\tau_{k}^{i}$ never exceeds a given integer $\tau \geq 0$. Thereby the exact gradient $\nabla f_{i}\left(x_{k}\right)$ is approximated by previous gradient components $\nabla f_{i}\left(x_{k-\tau_{k}^{i}}\right)$ no more than $\tau$ iterations before. We can rewrite (3) and (4) into the following subproblem:

$$
x_{k+1}=\arg \min _{x \in \mathbb{R}^{d}}\left\{h(x)+\left\langle g_{k}, x-x_{k}\right\rangle+\frac{1}{2 \alpha}\left\|x-x_{k}\right\|^{2}\right\} .
$$

Note that under the condition of no delays involved, i.e. $\tau_{k}^{i} \equiv 0$, we have $g_{k}=\sum \nabla f_{i}\left(x_{k}\right)$, which is exactly the classic FBS scheme.

On one hand, PIAG has been investigated in several works under the convex settings. [14 is the first to establish a global linear convergence rate of PIAG for strongly convex minimization, which guarantees that PIAG returns an $\varepsilon$-optimal solution after $\mathcal{O}\left(Q \tau^{2} \log _{2}(Q \tau) \log (1 / \varepsilon)\right)$ iterations, where $Q$ is the condition number. 3 . showed a global linear convergence rate in $\left\|x_{k}-x^{*}\right\|$ with complexity no more than $\mathcal{O}\left(\log (1 / \varepsilon) Q \tau^{2}\right)$; general distance functions are also involved in their analysis. Combining [14] and [3], 15] proposed stronger linear convergence rate that achieving an $\varepsilon$-optimal solution of the function values requires at most $O(Q \tau \log (1 / \varepsilon))$ iterations. [17] gave the global linear convergence of PIAG under several strictly weaker assumptions, novel variants with better convergence rate as well as an improved rate result under strongly convex condition. [18 proposed an accelerated globally linearly convergent scheme under quadratic growth condition, which combines the heavy ball method with Nesterov-like acceleration. 
On the other hand, there are a few studies of nonconvex FBS. A nonconvex nonsmooth version of FBS was analyzed in [2, which is involved in a fundamental approach under the Kurdyka-Łojasiewicz $(\mathrm{KL})$ condition. [5, 1 ] considers a structured functions of the type $L(x, y)=f(x)+Q(x, y)+g(x)$, with the case of nonconvex FBS included. Instead of using the KL conditions, this paper is consistent with a series of studies [8, 10, 4, 13, 12, using the proximal error bound condition, under which the recent work [16] gave local linear convergence results of an accelerated nonconvex proximal gradient method. The accelerated algorithm is exploiting historical information in essence, sharing the similar viewpoint with PIAG to an extent. Thus inspired by their work, we analyze the linear convergence of nonconvex PIAG with the proximal error bound condition.

Main contribution. In this study, we mainly focus on the convergence analysis of PIAG for minimizing a class of nonconvex problems, under the proximal error bound condition. First, we prove the sequence $\left\{x_{k}\right\}$ generated by PIAG is globally convergent to the stationary point set(Theorem 1(i)) of (1). Then, when we choose the stepsize $\alpha$ below a certain threshold, the objective function value sequence is proved to be R-linearly convergent to the function value at a certain stationary point(Theorem 1 (ii)). Finally, with the proved R-linearly convergent property of function value sequence, we show the iterate sequence $\left\{x_{k}\right\}$ generated by PIAG R-linearly converges to a certain stationary point.

The rest of the paper is structured as follows. Section 2 introduces notations and assumptions to be used. Section 3 gives the convergence analysis and section 4 concludes the paper.

\section{Notations \& Assumptions}

Throughout this paper, $d$-dimensional Euclidean space is denoted by $\mathbb{R}^{d}$ and its inner product is represented by $\langle\cdot, \cdot\rangle$. The $l_{2}$-norm is denoted by $\|\cdot\|$. For a nonempty closed set $\mathcal{C} \subset \mathbb{R}^{d}$, the distance from $x$ to $\mathcal{C}$ is represented by $\operatorname{dist}(x, \mathcal{C})$, where $\operatorname{dist}(x, \mathcal{C})=\inf _{y \in \mathcal{C}}\|x-y\|$. The domain of an extended-value function $h: \mathbb{R}^{d} \rightarrow[-\infty,+\infty]$ is defined as $\operatorname{dom} h=\left\{x \in \mathbb{R}^{d}, h(x)<+\infty\right\}$ and $h$ is said to be proper if $h$ is never equals $-\infty$ and $\operatorname{dom} h \neq \emptyset$. The gradient operator of a differentiable function is denoted by $\nabla$. The subdifferential of a proper lower closed convex function $h$ is defined as

$$
\partial h(x)=\left\{v \in \mathbb{R}^{d}: h(u)-h(x)-\langle v, u-x\rangle \geq 0, \quad \forall u \in \mathbb{R}^{d}\right\},
$$

where $\partial h(x)$ is always a closed convex set. The proximal operator of a proper closed function $h$ at $y \in \mathbb{R}^{d}$ is defined as

$$
\operatorname{Prox}_{h}(y)=\arg \min _{x \in \mathbb{R}^{d}}\left\{h(x)+\frac{1}{2}\|x-y\|^{2}\right\} .
$$

The sequence generated by PIAG is denoted by $\left\{x_{k}\right\} . \tilde{x}$ is said to be a stationary point of (1) if $0 \in \sum \nabla f_{i}(\tilde{x})+\partial h(\tilde{x})$. The set of all stationary points 
of (11) is denoted by $\mathcal{X}$. We say a sequence $\left\{x_{k}\right\}$ is R-linearly converges to $x^{*}$ if $\limsup _{k \rightarrow+\infty}\left\|x_{k}-x^{*}\right\|^{\frac{1}{k}}<1$. A function $f: \mathbb{R}^{d} \rightarrow \mathbb{R}$ is said to have a $L$-Lipschitz continuous gradient or to be $L$-smooth if

$$
\|\nabla f(x)-\nabla f(y)\| \leq L\|x-y\|, \quad \forall x, y \in \mathbb{R}^{d} .
$$

For the $L$-smooth function $f$, there always exists convex and gradient-Lipschitz continuous $f^{(j)}, j=1,2$ such that $f=f^{(1)}-f^{(2)}$. As illustrated in [16, one can choose $c>L$ and decompose $f$ in the following form:

$$
f=\underbrace{\left(f+\frac{c\|x\|^{2}}{2}\right)}_{f^{(1)}}-\underbrace{\frac{c\|x\|^{2}}{2}}_{f^{(2)}} .
$$

We list assumptions involved in this paper as follows.

A0. The objective function $F(x)$ in (1) is lower bounded.

A1. The decomposition $f_{i}=f_{i}^{(1)}-f_{i}^{(2)}$ exists for $i=1, \cdots, N$ such that $f_{i}^{(1)}$ is $L_{i}$-smooth and convex as well as $f_{i}^{(2)}$ is $l_{i}$-smooth and convex. Denote $L=\sum_{i=1}^{N} L_{i}$ and $l=\sum_{i=1}^{N} l_{i}$. Also assume $L_{i} \geq l_{i}$ and thus $f_{i}$ is $L_{i^{-}}$ smooth.

A2. The nonsmooth part $h: \mathbb{R}^{d} \rightarrow(-\infty, \infty]$ is proper, closed, convex and suqbdifferentiable everywhere in its effective domain, i.e., $\partial h(x) \neq \emptyset$ for all $x \in\left\{y \in \mathbb{R}^{d}: h(y)<\infty\right\}$.

A3. The time-varying delays $\tau_{k}^{i}$ are bounded; that is, there exists a nonnegative integer $\tau$ such that $\forall k \geq 1, i \in\{1,2, \cdots, N\}$, we have

$$
\tau_{k}^{i} \in\{0,1, \cdots, \tau\}
$$

where $\tau$ is named as the delay parameter.

The following two assumptions A4 and A5 are standard in the convergence analysis of several algorithms; see [8, 10, 4, 13, 12 and references therein.

A4. (Proximal Error Bound Condition) For any $\zeta \geq \inf _{x \in \mathbb{R}^{d}} F(x)$, there exist $\epsilon>0$ and $c_{0}>0$ such that

$$
\operatorname{dist}(x, \mathcal{X}) \leq c_{0}\left\|\operatorname{Prox}_{\frac{1}{L} h}\left(x-\frac{1}{L} \nabla f(x)\right)-x\right\|
$$

whenever $\left\|\operatorname{Prox}_{\frac{1}{L} g}\left(x-\frac{1}{L} \nabla f(x)\right)-x\right\|<\epsilon$ and $F(x) \leq \zeta$.

A5. There exists $\delta>0$, such that $\|x-y\| \geq \delta$ whenever $x, y \in \mathcal{X}, F(x) \neq F(y)$.

For simplicity, we further denote $f=\sum_{i=1}^{N} f_{i}, \bar{l}=\frac{l(\tau+1)}{2}, \bar{L}=\frac{L(\tau+1)}{2}$ and $\Delta_{k}=\sum_{j=k-\tau}^{k-1}\left\|x_{j+1}-x_{j}\right\|^{2}$. Without any loss of generality, let $x_{-k}=x_{0}$ for $k \geq 1$. 


\section{Convergence Analysis}

First, we give a sufficient descent property of PIAG for nonconvex minimization.

Lemma 1 With the assumptions A1-A3, the following statements for the problem (1) hold:

(i) For any $x \in \operatorname{dom} F$, we have the descent lemma that

$$
\begin{aligned}
F\left(x_{k+1}\right) & \leq F(x)+\left(\bar{l}+\frac{1}{2 \alpha}\right)\left\|x-x_{k}\right\|^{2}-\frac{1}{2 \alpha}\left\|x-x_{k+1}\right\|^{2} \\
& +\left(\bar{L}-\frac{1}{2 \alpha}\right)\left\|x_{k+1}-x_{k}\right\|^{2}+(\bar{l}+\bar{L}) \Delta_{k}, k \geq 0
\end{aligned}
$$

(ii) Consequently, we have the sufficient descent property that

$$
F\left(x_{k+1}\right) \leq F\left(x_{k}\right)+\left(\bar{L}-\frac{1}{\alpha}\right)\left\|x_{k+1}-x_{k}\right\|^{2}+(\bar{l}+\bar{L}) \Delta_{k}, k \geq 0 .
$$

Proof. By the convexity of $f_{i}^{(1)}$ and the $l_{i}$-smoothness of $f_{i}^{(2)}$, we have

$$
\begin{gathered}
f_{i}^{(1)}(x)+\left\langle\nabla f_{i}^{(1)}(x), y-x\right\rangle \leq f_{i}^{(1)}(y), \\
f_{i}^{(2)}(y) \leq f_{i}^{(2)}(x)+\left\langle\nabla f_{i}^{(2)}(x), y-x\right\rangle+\frac{l_{i}}{2}\|y-x\|^{2} .
\end{gathered}
$$

Adding up (14a) and (14b), using $f_{i}=f_{i}^{(1)}-f_{i}^{(2)}$, we obtain

$$
f_{i}(x)+\left\langle\nabla f_{i}(x), y-x\right\rangle \leq f_{i}(y)+\frac{l_{i}}{2}\|y-x\|^{2} .
$$

Due to the $L_{i}$-smoothness of $f_{i}$ and the inequality (15), we have

$$
\begin{aligned}
f_{i}\left(x_{k+1}\right) & \leq f_{i}\left(x_{x-\tau_{k}^{i}}\right)+\left\langle\nabla f_{i}\left(x_{k-\tau_{k}^{i}}\right), x_{k+1}-x_{k-\tau_{k}^{i}}\right\rangle+\frac{L_{i}}{2}\left\|x_{k+1}-x_{k-\tau_{k}^{i}}\right\|_{2}^{2} \\
& \leq f_{i}(x)+\left\langle\nabla f_{i}\left(x_{k-\tau_{k}^{i}}\right), x_{k+1}-x\right\rangle+\frac{l_{i}}{2}\left\|x-x_{k-\tau_{k}^{i}}\right\|_{2}^{2}+\frac{L_{i}}{2}\left\|x_{k+1}-x_{k-\tau_{k}^{i}}\right\|_{2}^{2} .
\end{aligned}
$$

Using the convexity of $\|\cdot\|^{2}$, we derive that

$$
\begin{aligned}
\sum_{i=1}^{N} \frac{l_{i}}{2}\left\|x-x_{k-\tau_{k}^{i}}\right\|^{2} & =\sum_{i=1}^{N} \frac{l_{i}}{2}\left\|\left(x-x_{k}\right)+\left(x_{k}-x_{k-1}\right)+\cdots+\left(x_{k-\tau_{k}^{i}+1}-x_{k-\tau_{k}^{i}}\right)\right\|^{2} \\
& \leq \sum_{i=1}^{N} \frac{l_{i}(\tau+1)}{2}\left(\left\|x-x_{k}\right\|^{2}+\sum_{j=k-\tau}^{k-1}\left\|x_{j+1}-x_{j}\right\|^{2}\right) \\
& =\bar{l}\left\|x-x_{k}\right\|^{2}+\bar{l} \Delta_{k} .
\end{aligned}
$$


Similarly,

$$
\sum_{i=1}^{N} \frac{L_{i}}{2}\left\|x_{k+1}-x_{k-\tau_{k}^{i}}\right\|^{2} \leq \bar{L}\left\|x_{k+1}-x_{k}\right\|^{2}+\bar{L} \Delta_{k} .
$$

With (17) and (18), the sum of (16) from $i=1$ to $N$ becomes

$$
\begin{aligned}
f\left(x_{k+1}\right) \leq & f(x)+\left\langle g_{k}, x_{k+1}-x\right\rangle+\bar{l}\left\|x-x_{k}\right\|^{2} \\
& +\bar{L}\left\|x_{k+1}-x_{k}\right\|^{2}+\bar{l} \Delta_{k}+\bar{L} \Delta_{k}
\end{aligned}
$$

where $g_{k}=\sum_{i=1}^{N} \nabla f\left(x_{k-\tau_{k}^{i}}\right)$. From the $\frac{1}{\alpha}-$ strongly convexity of subproblem (5), we have

$$
\begin{aligned}
\left\langle g_{k}, x_{k+1}-x\right\rangle \leq & h(x)-h\left(x_{k+1}\right)+\frac{1}{2 \alpha}\left\|x-x_{k}\right\|^{2} \\
& -\frac{1}{2 \alpha}\left\|x_{k+1}-x\right\|^{2}-\frac{1}{2 \alpha}\left\|x_{k+1}-x_{k}\right\|^{2}
\end{aligned}
$$

Plugging (20) into (19), we obtain

$$
\begin{aligned}
F\left(x_{k+1}\right) & \leq F(x)+\left(\bar{l}+\frac{1}{2 \alpha}\right)\left\|x-x_{k}\right\|^{2}-\frac{1}{2 \alpha}\left\|x-x_{k+1}\right\|^{2} \\
& +\left(\bar{L}-\frac{1}{2 \alpha}\right)\left\|x_{k+1}-x_{k}\right\|^{2}+(\bar{l}+\bar{L}) \Delta_{k} .
\end{aligned}
$$

Then the statement (i) holds. The statement (ii) follows from statement (i) by setting $x=x_{k}$.

Through the sufficient descent property of nonconvex PIAG, we give the following lemma to illustrate that for a fixed positive integer $M$, the sequence $\left\{x_{k}\right\}$ satisfies

$$
\lim _{k \rightarrow \infty}\left\|x_{k+M}-x_{k}\right\|=\lim _{k \rightarrow \infty} \sum_{j=k}^{j=k+M}\left\|x_{j+1}-x_{j}\right\| \rightarrow 0 .
$$

Lemma 2 Assume A0-A3 hold. If stepsize $\alpha<\frac{1}{\bar{L}+\tau(\bar{l}+\bar{L})}$, then the following statements hold:

(i) $F\left(x_{k}\right)$ is bounded;

(ii) $\sum_{k=0}^{\infty}\left\|x_{k+1}-x_{k}\right\|^{2}<+\infty$ 
Proof. From Lemma 1(ii), for arbitrary positive integers $k_{1}<k_{2}$, summing up (13) from $k=k_{1}$ to $k_{2}-1$ yields

$$
\begin{aligned}
F\left(x_{k_{2}}\right) & \leq F\left(x_{k_{1}}\right)+\left(\bar{L}-\frac{1}{\alpha}\right) \sum_{k=k_{1}}^{k_{2}-1}\left\|x_{k+1}-x_{k}\right\|^{2}+(\bar{l}+\bar{L}) \sum_{k=k_{1}}^{k_{2}-1} \Delta_{k} \\
& \leq F\left(x_{k_{1}}\right)+\left(\bar{L}-\frac{1}{\alpha}\right) \sum_{k=k_{1}}^{k_{2}-1}\left\|x_{k+1}-x_{k}\right\|^{2}+\tau(\bar{l}+\bar{L}) \sum_{k=k_{1}-\tau}^{k_{2}-1}\left\|x_{k+1}-x_{k}\right\|^{2} \\
& \leq F\left(x_{k_{1}}\right)+(\bar{L}+\tau(\bar{l}+\bar{L})) \sum_{k=k_{1}-\tau}^{k_{2}-1}\left\|x_{k+1}-x_{k}\right\|^{2}-\frac{1}{\alpha} \sum_{k=k_{1}}^{k_{2}-1}\left\|x_{k+1}-x_{k}\right\|^{2} .
\end{aligned}
$$

Setting $k_{1}=0$ and $k_{2}=K+1$, we obtain

$$
F\left(x_{K+1}\right) \leq F\left(x_{0}\right)+\left(\bar{L}-\frac{1}{\alpha}+\tau(\bar{l}+\bar{L})\right) \sum_{k=0}^{K}\left\|x_{k+1}-x_{k}\right\|^{2},
$$

which indicates that $F\left(x_{k}\right)$ is bounded from above if $\alpha<\frac{1}{\bar{L}+\tau(l+\bar{L})}$. With A0 that inf $F>-\infty$ holds, (24) implies

$$
\sum_{k=0}^{K}\left\|x_{k+1}-x_{k}\right\|^{2} \leq \frac{F\left(x_{0}\right)-F\left(x_{K+1}\right)}{\frac{1}{\alpha}-\tau(\bar{l}+\bar{L})-\bar{L}}
$$

The inequality holds as $K \rightarrow \infty$. Thus statement (ii) is proved.

Lemma 3 Assume that A0-A3 hold and $\alpha<\frac{1}{\bar{L}+\tau(\bar{l}+\bar{L})}$. Then, any accumulation point of $\left\{x_{k}\right\}$ is a stationary point of $F$.

Proof. Let $\bar{x}$ be an accumulation point. Then there exists a subsequence $\left\{x_{k_{i}}\right\}$ such that $\lim _{i \rightarrow \infty} x_{k_{i}}=\bar{x}$. Using the first-order optimality condition of subproblem (5), we have

$$
-\frac{1}{\alpha}\left(x_{k_{i}+1}-x_{k_{i}}\right) \in \sum_{j=1}^{N} \nabla f_{j}\left(x_{k_{i}-\tau_{k_{i}}^{j}}\right)+\partial h\left(x_{k_{i}+1}\right) .
$$

Invoking Lemma 2(ii), for an arbitrary fixed integer $I \in\{0,1, \cdots, \tau\}$, we have

$$
\lim _{i \rightarrow+\infty} x_{k_{i}-I}=\lim _{i \rightarrow+\infty} x_{k_{i}+1}=\bar{x}
$$

which implies $\lim _{i \rightarrow+\infty} x_{k_{i}-\tau_{k_{i}}^{j}}=\bar{x}$ since $0 \leq \tau_{k_{i}}^{j} \leq \tau$ for any $j \in\{1,2, \cdots, N\}$. Consequently, due to continuity of $\nabla f$ and closedness of $\partial h$, (26) implies $0 \in$ $\nabla f(\bar{x})+\partial h(\bar{x})$. 
Lemma 4 Assume that A0-A3 hold and $\alpha<\frac{1}{L+\tau(l+L)}$. Let $\Omega$ be the set of accumulation points of the sequence $\left\{x_{k}\right\}$ generated by PIAG. Then $\zeta=$ $\lim _{k \rightarrow \infty} F\left(x_{k}\right)$ exists and $F \equiv \zeta$ on $\Omega$.

Proof. The fact that $F\left(x_{k}\right)$ is bounded has been shown in Lemma 2(i). Thus if the limit of $F\left(x_{k}\right)$ does not exist, then there are two subsequences $\left\{x_{s_{i}^{1}}\right\}$ and $\left\{x_{s_{i}^{2}}\right\}$ of $\left\{x_{k}\right\}$ such that $F\left(x_{s_{i}^{1}}\right) \rightarrow F_{1}$ and $F\left(x_{s_{i}^{2}}\right) \rightarrow F_{2}$. Without loss of generality, suppose $F_{1}>F_{2}$.

First, due to Lemma 2(ii), there exists a sufficiently large positive integer $K$ such that

$$
(\bar{L}+\tau(\bar{l}+\bar{L})) \sum_{j=K-\tau}^{\infty}\left\|x_{j+1}-x_{j}\right\|^{2}<\frac{F_{1}-F_{2}}{3} .
$$

Second, from (23) we can find two sufficiently large subscript indexes $S_{1} \in$ $\left\{s_{i}^{1}\right\}$ and $S_{2} \in\left\{s_{i}^{2}\right\}$ such that $S_{1}>S_{2}>K$ and satisfy

$$
\begin{gathered}
F\left(x_{S_{1}}\right)-F\left(x_{S_{2}}\right) \leq(\bar{L}+\tau(\bar{l}+\bar{L})) \sum_{j=S_{2}-\tau}^{S_{1}-1}\left\|x_{j+1}-x_{j}\right\|^{2} \\
<\frac{F_{1}-F_{2}}{3}, \\
F_{1}-F\left(x_{S_{1}}\right)<\frac{F_{1}-F_{2}}{3} \\
F\left(x_{S_{2}}\right)-F_{2}<\frac{F_{1}-F_{2}}{3} .
\end{gathered}
$$

The sum of (29), (30) and (31) derives the contradiction $F_{1}-F_{2}<F_{1}-F_{2}$. Thus $\lim F\left(x_{k}\right)$ must exist. Denote the limit by $\zeta$.

If $\Omega=\emptyset$, the result is trivially true. Otherwise, $\forall \hat{x} \in \Omega$, suppose a subsequence $x_{k_{i}} \rightarrow \hat{x}$. Due to the lower semi-continuity of $F$, we have

$$
F(\hat{x}) \leq \liminf F\left(x_{k_{i}}\right)=\zeta .
$$

On the other hand, since $x_{k_{i}+1}$ is the minimizer of

$$
h(x)+\left\langle g_{k_{i}}, x-x_{k_{i}}\right\rangle+\frac{1}{2 \alpha}\left\|x-x_{k_{i}}\right\|^{2},
$$

we have

$$
\begin{aligned}
& f\left(x_{k_{i}+1}\right)+h\left(x_{k_{i}+1}\right)+\left\langle g_{k_{i}}, x_{k_{i}+1}-\hat{x}\right\rangle+\frac{1}{2 \alpha}\left\|x_{k_{i}+1}-x_{k_{i}}\right\|^{2} \\
\leq & f\left(x_{k_{i}+1}\right)+h(\hat{x})+\frac{1}{2 \alpha}\left\|\hat{x}-x_{k_{i}}\right\|^{2} .
\end{aligned}
$$

Letting $i \rightarrow \infty$, we obtain

$$
\limsup F\left(x_{k_{i}}\right) \leq F(\hat{x}) .
$$

Along with (32), the equality $F(\hat{x}) \equiv \zeta$ holds for all $\hat{x} \in \Omega$. 
Furthermore, if we assume $F$ is level bounded, since we already know $F\left(x_{k}\right)$ is upper bounded from Lemma 2, then the sequence $\left\{x_{k}\right\}$ is also bounded which $\operatorname{implies} \Omega \neq \emptyset$ in 1 emma 3 implies that .

The stepsize $\alpha$ is required to be small in previous lemmas but is undetermined for now. We might require a sufficiently small $\alpha$ in PIAG to guarantee convergence. The A4 for a fixed stepsize $\alpha=\frac{1}{L}$ seems inadequate for later proof. Therefore, we need a variant of A4 with stepsizes smaller than $\frac{1}{L}$. To make the fact explicit, we display the result in the following two lemmas.

Lemma $5 \sqrt[7]{7}$, lemma 2] Suppose that function $h: \mathbb{R}^{d} \rightarrow \mathbb{R}$ satisfies A2 and $f: \mathbb{R}^{d} \rightarrow \mathbb{R}$ is differentiable on $\mathbb{R}^{d}$. Then, $\forall x \in \operatorname{dom} h$ and real numbers $t \geq t^{\prime}>0$, we have

$$
\frac{1}{t}\left\|\operatorname{Prox}_{t h}(x-t \nabla f(x))-x\right\| \leq \frac{1}{t^{\prime}}\left\|\operatorname{Prox}_{t h}\left(x-t^{\prime} \nabla f(x)\right)-x\right\| .
$$

Lemma 6 If $A 4$ holds with $\epsilon>0, c_{0}>0$ and $\zeta \geq \inf _{x \in \mathbb{R}^{d}} F(x)$, then

$$
\operatorname{dist}(x, \mathcal{X}) \leq \frac{c_{0}}{\alpha L}\left\|\operatorname{Prox}_{\alpha h}(x-\alpha \nabla f(x))-x\right\|,
$$

whenever $0<\alpha \leq \frac{1}{L}$, $\left\|\operatorname{Prox}_{\alpha h}(x-\alpha \nabla f(x))-x\right\|<\alpha L \epsilon$ and $F(x) \leq \zeta$.

Proof. For $\alpha \in\left(0, \frac{1}{L}\right]$, if $x$ satisfies $\left\|\operatorname{Prox}_{\alpha h}(x-\alpha \nabla f(x))-x\right\|<\alpha L \epsilon$ and $F(x) \leq \zeta$, invoking Lemma 5, then we have

$$
\left\|\operatorname{Prox}_{\frac{1}{L} h}\left(x-\frac{1}{L} \nabla f(x)\right)-x\right\| \leq \frac{1}{\alpha L}\left\|\operatorname{Prox}_{\alpha h}(x-\alpha \nabla f(x))-x\right\|<\epsilon .
$$

Thus A4 gives

$$
\begin{aligned}
\operatorname{dist}(x, \mathcal{X}) & \leq c_{0}\left\|\operatorname{Prox}_{\frac{1}{L} h}\left(x-\frac{1}{L} \nabla f(x)\right)-x\right\| \\
& \leq \frac{c_{0}}{\alpha L}\left\|\operatorname{Prox}_{\alpha h}(x-\alpha \nabla f(x))-x\right\|,
\end{aligned}
$$

which is just (38).

Before proposing the final results, we need the following key lemma for revealing the linear convergence.

Lemma 7 [3] Assume that the non-negative sequences $\left\{V_{k}\right\}$ and $\left\{\omega_{k}\right\}$ satisfy the following inequality:

$$
V_{k+1} \leq a V_{k}-b \omega_{k}+c \sum_{j=k-k_{0}}^{k} \omega_{j},
$$

for some real numbers $a \in(0,1)$ and $b, c \geq 0$, and some positive integer $k_{0}$. Also Assume that $\omega_{k}=0$ for $k<0$, and that the following holds:

$$
\frac{c}{1-a} \frac{1-a^{k_{0}+1}}{a^{k_{0}}} \leq b .
$$

Then, $V_{k} \leq a^{k} V_{0}$ for all $k \geq 0$. 
Theorem 1 For arbitrary sequence $\left\{x_{k}\right\}$ generated by PIAG, with the assumption A0-A5, for all sufficiently small stepsize $\alpha \geq 0$, the following statements hold:

(i) $\operatorname{dist}\left(x_{k}, \mathcal{X}\right) \rightarrow 0$;

(ii) $F\left(x_{k}\right)$ is R-linearly convergent.

Proof. We derive that

$$
\begin{aligned}
& \left\|\operatorname{Prox}_{\alpha h}\left(x_{k}-\alpha \nabla f\left(x_{k}\right)\right)-x_{k}\right\| \\
\leq & \left\|\operatorname{Prox}_{\alpha h}\left(x_{k}-\alpha \nabla f\left(x_{k}\right)\right)-\operatorname{Prox}_{\alpha h}\left(x_{k}-\alpha g_{k}\right)\right\|+\left\|\operatorname{Prox}_{\alpha h}\left(x_{k}-\alpha g_{k}\right)-x_{k}\right\| \\
\leq & \alpha \sum\left\|\nabla f_{i}\left(x_{k}\right)-\nabla f_{i}\left(x_{k-\tau_{k}^{i}}\right)\right\|+\left\|x_{k+1}-x_{k}\right\| \\
\leq & \alpha \sum L_{i}\left\|x_{k}-x_{k-\tau_{k}^{i}}\right\|+\left\|x_{k+1}-x_{k}\right\| \\
\leq & \sqrt{(\alpha L+1)\left(\alpha \sum_{i=1}^{N} L_{i}\left\|x_{k}-x_{k-\tau_{k}^{i}}\right\|^{2}+\left\|x_{k+1}-x_{k}\right\|^{2}\right)} \\
\leq & \sqrt{(a L+1)\left(\alpha \tau L \sum_{j=k-\tau}^{k-1}\left\|x_{j+1}-x_{j}\right\|^{2}+\left\|x_{k+1}-x_{k}\right\|^{2}\right)}
\end{aligned}
$$

In addition, we require

$$
\alpha<\min \left(\frac{1}{\bar{L}+\tau(\bar{l}+\bar{L})}, \frac{1}{L}\right)
$$

With Lemma 2(ii), the inequality (44) implies

$$
\left\|\operatorname{Prox}_{\alpha h}\left(x_{k}-\alpha \nabla f\left(x_{k}\right)\right)-x_{k}\right\| \rightarrow 0
$$

In addition to (46), Lemma 4 implies that $F\left(x_{k}\right)$ is bounded, so we can conclude there exists a large enough positive number $K_{\alpha}$ relevant to $\alpha$ such that the inequality (38) could apply whenever $k>K_{\alpha}$. Therefore for each $k>K_{\alpha}$, using Assumption A4 and inequality (44) we have

$$
\operatorname{dist}\left(x_{k}, \mathcal{X}\right)^{2} \leq\left(\frac{c_{0}}{\alpha L}\right)^{2}(a L+1)\left(\alpha \tau L \sum_{j=k-\tau}^{k-1}\left\|x_{j+1}-x_{j}\right\|^{2}+\left\|x_{k+1}-x_{k}\right\|^{2}\right)
$$

which readily leads to $\operatorname{dist}\left(x_{k}, \mathcal{X}\right) \rightarrow 0$. Thus the statement (i) is proved. 
Setting $x=\bar{x}_{k}$ in (16), where $\bar{x}_{k}$ represents a projection of $x_{k}$ onto $\mathcal{X}$ then we obtain

$$
\begin{aligned}
F\left(x_{k+1}\right) & \leq F\left(\bar{x}_{k}\right)+\left(\frac{l(\tau+1)}{2}+\frac{1}{2 \alpha}\right) \operatorname{dist}\left(x_{k}, \mathcal{X}\right)^{2}+\left(\frac{L(\tau+1)}{2}-\frac{1}{2 \alpha}\right)\left\|x_{k+1}-x_{k}\right\|^{2} \\
& +\frac{(l+L)(\tau+1)}{2} \sum_{j=k-\tau}^{k-1}\left\|x_{j+1}-x_{j}\right\|^{2} \\
& \leq F\left(\bar{x}_{k}\right)+\left(\frac{L(\tau+1)}{2}-\frac{1}{2 \alpha}+\left(\frac{l(\tau+1)}{2}+\frac{1}{2 \alpha}\right)\left(\frac{c_{0}}{\alpha L}\right)^{2}(\alpha L+1)\right)\left\|x_{k+1}-x_{k}\right\|^{2} \\
& +\left[\frac{(l+L)(\tau+1)}{2}+\left(\frac{l(\tau+1)}{2}+\frac{1}{2 \alpha}\right)\left(\frac{c_{0}}{\alpha L}\right)^{2}(\alpha L+1) \alpha \tau L\right] \sum_{j=k-\tau}^{k-1}\left\|x_{j+1}-x_{j}\right\|^{2}
\end{aligned}
$$

Since $\left\|x_{k}-x_{k+1}\right\| \rightarrow 0$ and $\operatorname{dist}\left(x_{k}, \mathcal{X}\right) \rightarrow 0$, nothing that

$$
\left\|\bar{x}_{k}-\bar{x}_{k+1}\right\| \leq \operatorname{dist}\left(x_{k}, \mathcal{X}\right)+\left\|x_{k}-x_{k+1}\right\|+\operatorname{dist}\left(x_{k+1}, \mathcal{X}\right)
$$

we have $\left\|\bar{x}_{k}-\bar{x}_{k+1}\right\| \rightarrow 0$. With A5, $F\left(\bar{x}_{k}\right) \equiv \zeta$ holds for some constant $\zeta$ for all sufficiently large $k$. Without loss of generality, we suppose $F\left(\bar{x}_{k}\right)=\zeta$ for $k \geq K_{\alpha}$. Relax $\alpha$ to $\frac{1}{L}$ and rewrite (13) to (49), $\alpha^{2} \times($ (47) to (50) in a simplified way as follow:

$$
\begin{aligned}
& F\left(x_{k+1}\right) \leq F\left(x_{k}\right)+\left(C_{1}-\frac{1}{\alpha}\right)\left\|x_{k+1}-x_{k}\right\|^{2}+C_{2} \sum_{j=k-\tau}^{k-1}\left\|x_{j+1}-x_{j}\right\|^{2}, \\
& \alpha^{2} F\left(x_{k+1}\right) \leq \alpha^{2} \zeta+\left(C_{3}+\frac{c_{0}^{2}}{L^{2}} \frac{1}{2 \alpha}\right)\left\|x_{k+1}-x_{k}\right\|^{2}+C_{4} \sum_{j=k-\tau}^{k-1}\left\|x_{j+1}-x_{j}\right\|^{2},
\end{aligned}
$$

where constants $C_{i}$ are independent with $\alpha$. Denote $H\left(x_{k}\right):=F\left(x_{k}\right)-\zeta$. Therefore, (49) $+\frac{L^{2}}{c_{0}^{2}}(150)$ leads to

$$
\left(1+\frac{L^{2}}{c_{0}^{2}} \alpha^{2}\right) H\left(x_{k+1}\right) \leq H\left(x_{k}\right)+\left(C_{5}-\frac{1}{2 \alpha}\right)\left\|x_{k+1}-x_{k}\right\|^{2}+C_{6} \sum_{j=k-\tau}^{k-1}\left\|x_{j+1}-x_{j}\right\|^{2}
$$

Actually, from the inequality above, one can directly conclude that for all sufficiently small $\alpha$, Lemma 7 could always be employed to obtain the linear convergence of $H\left(x_{k}\right) \rightarrow 0$. The remaining trivial piece is to give an explicit range of $\alpha$. All constants are listed as follows (a verifying Walfram Mathemat- 
ica script is available online https://www.deepinfar.cn/piag):

$$
\begin{aligned}
C_{1} & =\frac{L(\tau+1)}{2}, C_{2}=\frac{(l+L)(\tau+1)}{2}, \\
C_{3} & =\frac{c_{0}^{2}(2 l(\tau+1)+L)+L \tau}{2 L^{2}}, \\
C_{4} & =\frac{(l+L)(1+\tau)+2 \tau(l+L+l \tau) c_{0}^{2}}{2 L^{2}}, \\
C_{5} & =l+L+l \tau+\frac{L \tau}{2}+\frac{L \tau}{2 c_{0}^{2}}, \\
C_{6} & =\frac{1}{2}\left(\frac{(\tau+1)(l+L)}{c_{0}^{2}}+2 l \tau^{2}+3 l \tau+l+3 L \tau+L\right) .
\end{aligned}
$$

With consistent notations in Lemma 7, let $V_{k}=\left(1+\frac{L^{2}}{c_{0}^{2}} \alpha^{2}\right) H\left(x_{k}\right), a=$ $\left(1+\frac{L^{2}}{c_{0}^{2}} \alpha^{2}\right)^{-1}, b=\frac{1}{2 \alpha}-C_{5}, k_{0}=\tau$ and $c=C_{6}$. Note $\alpha \leq \frac{1}{L}$, then $a \geq$ $\left(1+\frac{1}{c_{0}^{2}}\right)^{-1}$ and the left side of (43) is bounded by

$$
\frac{c}{1-a} \frac{1-a^{k_{0}+1}}{a^{k_{0}}}=c \sum_{j=0}^{k_{0}} a^{-j} \leq C_{6}\left(1+\tau\left(1+\frac{1}{c_{0}^{2}}\right)^{\tau}\right) \triangleq C_{7},
$$

which implies that when $\alpha \leq \frac{1}{2 C_{5}+2 C_{7}}$, we have

$$
\frac{c}{1-a} \frac{1-a^{k_{0}+1}}{a^{k_{0}}} \leq C_{7} \leq \frac{1}{2 \alpha}-C_{5}=b .
$$

The inequality (43) in Lemma 7 holds. Thus let

$$
\alpha=C_{8}=\min \left(\frac{1}{\bar{L}+\tau(\bar{l}+\bar{L})}, \frac{1}{2 C_{5}+2 C_{7}}, \frac{1}{L}\right)
$$

and we have

$$
F\left(x_{k}\right)-\zeta \leq\left(1+\frac{L^{2}}{c_{0}^{2}} \frac{1}{C_{8}^{2}}\right)^{K_{\alpha}-k}\left(F\left(x_{K_{\alpha}}\right)-\zeta\right), \quad k \geq K_{\alpha}
$$

Moreover, we claim that the path of $\left\{x_{k}\right\}$ has finite length and $\left\{x_{k}\right\} \mathrm{R}$ linearly converges to some stationary point $\tilde{x}$. First, we prove the following lemma.

Lemma 8 Let $\left\{a_{k}\right\},\left\{b_{k}\right\}$ be positive sequences where $b_{k}=b_{0} q^{k}$ for a real number $q \in(0,1)$. If the inequality

$$
a_{k} \leq b_{k}+\frac{c}{\tau}\left(a_{k-1}+a_{k-2}+\cdots+a_{k-\tau}\right)
$$

holds for $k \geq \tau$ where $\tau$ is a given positive integer and $0<c<1$, then $\left\{a_{k}\right\}$ is $R$-linearly convergent to zero. 
Proof. We consider a characteristic polynomial

$$
P(x)=x^{\tau}-\frac{c}{\tau} x^{\tau-1}-\frac{c}{\tau} x^{\tau-2}-\cdots-\frac{c}{\tau} .
$$

Since $P(c) \leq 0$ and $P(1)=1-c>0$, letting a root of $P(x)$ in $[c, 1)$ be denoted by $p$, then we have the following inequalities,

$$
\begin{aligned}
a_{k} & \leq b_{k}+\frac{c}{\tau}\left(a_{k-1}+a_{k-2}+\cdots+a_{k-\tau}\right), \\
p a_{k-1} & \leq p b_{k-1}+\frac{c}{\tau}\left(p a_{k-2}+p a_{k-3}+\cdots+p a_{k-\tau-1}\right), \\
p^{2} a_{k-2} & \leq p^{2} b_{k-2}+\frac{c}{\tau}\left(p^{2} a_{k-3}+p^{2} a_{k-4}+\cdots+p^{2} a_{k-\tau-2}\right), \\
& \ldots \\
p^{k-\tau} a_{\tau} & \leq p^{k-\tau} b_{\tau}+\frac{c}{\tau}\left(p^{k-\tau} a_{\tau-1}+p^{k-\tau} a_{\tau-2}+\cdots+p^{k-\tau} a_{0}\right) .
\end{aligned}
$$

Adding them up, with proper relaxing, we have

$$
\begin{aligned}
a_{k} & \leq \sum_{i=0}^{k} p^{k-i} b_{i}+c p^{k-2 \tau+1} \sum_{i=0}^{\tau-1} a_{i} \\
& =b_{0} p^{k} \sum_{i=0}^{k}\left(\frac{q}{p}\right)^{i}+c p^{k-2 \tau+1} \sum_{i=0}^{\tau-1} a_{i} .
\end{aligned}
$$

Perform limit superior on both sides, we have

$$
\limsup _{k \rightarrow+\infty}\left|a_{k}\right|^{\frac{1}{k}} \leq \max (p, q)<1 .
$$

Thus $\left\{a_{k}\right\}$ is R-linearly convergent to 0 .

Theorem 2 Suppose conditions of Theorem 1 are satisfied. Then we have

(i) $\sum_{i=0}^{\infty}\left\|x_{i+1}-x_{i}\right\|<+\infty$,

(ii) $\left\{x_{k}\right\}$ is R-linearly convergent.

Proof. Rewrite the inequality (49) as

$$
\left\|x_{k+1}-x_{k}\right\|^{2} \leq \frac{C_{2}}{\frac{1}{\alpha}-C_{1}}\left(F\left(x_{k}\right)-F\left(x_{k+1}\right)\right)+\frac{C_{2}}{\frac{1}{\alpha}-C_{1}} \sum_{j=k-\tau}^{k-1}\left\|x_{j+1}-x_{j}\right\|^{2} .
$$

Since the first term of the right sides is proved to be R-linearly convergent and the coefficient of the second term satisfies

$$
\frac{C_{2}}{\frac{1}{\alpha}-C_{1}}=\frac{C_{2}}{2 C_{5}+2 C_{7}-C_{1}}<\frac{C_{2}}{2 C_{5}+2 \tau C_{6}-C_{1}}<\frac{1}{\tau},
$$


thus Lemma 8 implies that $\left\|x_{k+1}-x_{k}\right\| \leq r_{0} \cdot r^{k}$ for some $0<r<1, r_{0}>0$, which illustrates the statement (i). Consequently $\left\{x_{k}\right\}$ is a Cauchy sequence and then it converges to a point $\tilde{x} \in \mathcal{X}$. Finally we have

$$
\left\|x_{k}-\tilde{x}\right\| \leq \sum_{j=k}^{\infty}\left\|x_{j}-x_{j+1}\right\| \leq \frac{r_{0} \cdot r^{k}}{1-r},
$$

which implies that $x_{k}$ R-linearly converges to $\tilde{x}$.

\section{Conclusion}

In this paper, we analyze the convergence of PIAG for nonconvex minimization. First of all, we give the sufficient descent property of PIAG in nonconvex cases. Under the proximal error bound condition, we prove that the generated sequence $\left\{x_{k}\right\}$ is convergent to the stationary point set. Then, we show $\left\{F\left(x_{k}\right)\right\}$ is R-linearly convergent and that $\left\{x_{k}\right\}$ R-linearly converges to a stationary point when the stepsize $\alpha$ is under some positive constant. Finally, we note that even with the delay parameter $\tau$ vanishing, our theoretical convergence rate is far from being tight, which deserves further study.

\section{References}

1. Hédy Attouch, Jérôme Bolte, Patrick Redont, and Antoine Soubeyran. Proximal alternating minimization and projection methods for nonconvex problems: An approach based on the kurdyka-łojasiewicz inequality. Mathematics of Operations Research, 35(2):438-457, 2010.

2. Hedy Attouch, Jérôme Bolte, and Benar Fux Svaiter. Convergence of descent methods for semi-algebraic and tame problems: proximal algorithms, forward-backward splitting, and regularized gauss-seidel methods. Mathematical Programming, 137(1-2):91-129, 2013.

3. Arda Aytekin, Hamid Reza Feyzmahdavian, and Mikael Johansson. Analysis and implementation of an asynchronous optimization algorithm for the parameter server. arXiv preprint arXiv:1610.05507, 2016.

4. Amir Beck and Marc Teboulle. A linearly convergent dual-based gradient projection algorithm for quadratically constrained convex minimization. Mathematics of Operations Research, 31(2):398-417, 2006.

5. Jérôme Bolte, Shoham Sabach, and Marc Teboulle. Proximal alternating linearized minimization for nonconvex and nonsmooth problems. Mathematical Programming, 146(1-2):459-494, 2014.

6. Patrick L Combettes and Valérie R Wajs. Signal recovery by proximal forward-backward splitting. Multiscale Modeling \& Simulation, 4(4):1168-1200, 2005.

7. Yuanyuan Huang and Yunda Dong. New properties of forwardbackward splitting and a practical proximal-descent algorithm. Applied Mathematics and Computation, 237:6068, 2014.

8. Zhi-Quan Luo and Paul Tseng. On the linear convergence of descent methods for convex essentially smooth minimization. SIAM Journal on Control and Optimization, 30(2):408-425, 1992.

9. Zhi-Quan Luo and Paul Tseng. Error bounds and convergence analysis of feasible descent methods: a general approach. Annals of Operations Research, 46(1):157-178, 1993 
10. Zhi-Quan Luo and Paul Tseng. On the convergence rate of dual ascent methods for linearly constrained convex minimization. Mathematics of Operations Research, 18(4):846867, 1993.

11. Paul Tseng. Approximation accuracy, gradient methods, and error bound for structured convex optimization. Mathematical Programming, 125(2):263-295, 2010.

12. Paul Tseng and Sangwoon Yun. A coordinate gradient descent method for nonsmooth separable minimization. Mathematical Programming, 117(1-2):387-423, 2009.

13. Paul Tseng and Sangwoon Yun. A coordinate gradient descent method for linearly constrained smooth optimization and support vector machines training. Computational Optimization and Applications, 47(2):179-206, 2010.

14. Nuri Denizcan Vanli, Mert Gurbuzbalaban, and Asu Ozdaglar. Global convergence rate of proximal incremental aggregated gradient methods. arXiv preprint arXiv:1608.01713, 2016

15. Nuri Denizcan Vanli, Mert Gurbuzbalaban, and Asu Ozdaglar. A stronger convergence result on the proximal incremental aggregated gradient method. arXiv preprint arXiv:1611.08022, 2016.

16. Bo Wen, Xiaojun Chen, and Ting Kei Pong. Linear convergence of proximal gradient algorithm with extrapolation for a class of nonconvex nonsmooth minimization problems. SIAM Journal on Optimization, 27(1):124-145, 2017.

17. Hui Zhang. Linear convergence of the proximal incremental aggregated gradient method under quadratic growth condition. arXiv preprint arXiv:1702.08166, 2017.

18. Xiaoya Zhang, Wei Peng, Hui Zhang, and Wei Zhu. Inertial proximal incremental aggregated gradient method. arXiv preprint arXiv:1712.00984, 2017. 